\title{
LA RECREACIÓN EN LA ESCUELA: UN CAMPO DE EXPLORACIÓN Y GESTIÓN DE LA IMAGINACIÓN ${ }^{1}$

\author{
THE RECREATION AT SCHOOL: AN EXPLORATION AND MANAGEMENT \\ FIELD OF IMAGINATION
}

\author{
Joselín Acosta ${ }^{2}$ \\ Pompilio Gutiérrez ${ }^{3}$ \\ Astrid Bibiana Rodríguez ${ }^{4}$
}

\section{Resumen}

La sociedad contemporánea exige de la escuela mediaciones pedagógicas que permitan a los educandos herramientas y competencias para entender de forma reflexiva y critica un mundo nunca antes imaginado. Las lógicas racionalizantes, que antes ocupaba cierta preponderancia en los modos de comprensión, ya no son suficientes para explicar la emergencia permanente de conocimiento y una inusual irregularidad en los acontecimientos que, en otro tiempo, se percibían con mayor estabilidad. Todo se trastoca, cambia, se fisura en el mundo actual.

Si se acepta esta idea de movilidad permanente, la recreación como campo diferenciado de saber en la escuela ha de ser explorada en sus lenguajes alterativos, en la expansión de lo simbólico, en inusuales y variados empalabramientos con la realidad y, por supuesto, en su expresa intención de transformar, como se dijo antes, la preponderancia de ciertos saberes que ya no son suficientes, aunque necesarios, para transitar por un mundo cada vez más exigente a la imaginación humana. Es desde esta perspectiva de apertura y gestión a la imaginación que la recreación en la escuela puede hacer de lo familiar algo extraño y posible para ser nuevamente explorado, re-escrito y contado de modo distinto a las actuales y futuras generaciones.

Palabras clave: recreación, sociedad contemporánea, creatividad e imaginación, escuela y pedagogía.

\section{Abstract}

The contemporary society demands of the school pedagogic mediations that allow tools and competitions to the students to deal on reflexive and critic way a world never before imagined. The racional logics, that before was occupying certain prevalence in the ways to comprehension, are not already sufficient to explain the permanent emergency of knowledge and an unusual irregularity in the events that, in another time, were perceived by most stability. Everything disrupt, it changes, fissure in the current world.

If there is accepted this idea of permanent mobility, the recreation like differentiated field of knowing in the school has to be explored in his alterative languages, in the expansion of the symbolic thing, in unusual and varied in-wordly with the reality and, certainly, in his express intention of transforming, as was said before, the prevalence of certain knowledges that are not already sufficient, though necessary, to pass along a world increasingly demandingly to the human imagination. It is from this

\footnotetext{
${ }^{1}$ Este texto corresponde a una reflexión del proyecto de investigación Pedagogía y didáctica de la recreación en la escuela, que pertenece a la línea de Investigación: Cuerpo, lúdica y sociedad. Categoría D Colciencias. Director Jhon Jairo Uribe.

${ }^{2}$ Profesor ocasional Universidad Pedagógica Nacional. Profesor Licenciatura en Recreación joselin_acosta@yahoo.com

${ }^{3}$ Profesor ocasional Universidad Pedagógica Nacional. Licenciatura en Recreación piliox@hotmail.com

${ }^{4}$ Profesora de Planta de la Universidad Pedagógica Nacional. Coordinadora Licenciatura en Recreación abrodriguez@pedagogica.edu.co.
}

Universidad Pedagógica $\mathrm{N}$ acional 
perspective of opening and management to the imagination that the recreation in the school can do of the slightly strange and possible familiar thing to be explored again, rewritten and counted in a way different from the current and future generations.

Key words: recreation, contemporary society, creativity and imagination, school and pedagogy.

Fecha de recepción: 12 de Septiembre de 2010

Fecha de aprobación: 1 de Noviembre de 2010

\section{Introducción}

La época actual viene experimentando vertiginosos avances en variados campos del conocimiento. Los movimientos científicos, tecnológicos, en el arte, la literatura y, en general, en las maneras como el sujeto se relaciona con el mundo se caracteriza por la presencia continuas caducidades en los modos de comprensión y actuación humana.

En este sentido, por ejemplo, la sociedad de información y consumo transformó por completo las relaciones de producción del artesano que, en otro tiempo, se presumía dueño de su arte. Se "progresó" a un arte mecanizado y a un artesano sometido al tiempo de la producción: sujeto-objeto de un hacer mercantilizado. Así, el homo faber, como productor de una vida en común y quien era el juez del trabajo y la práctica material fue desplazado, en palabras de Arendt (2008), a un animal laborans que se asemeja más a una bestia de carga, un "siervo condenado a la rutina" y la velocidad del progreso que, en muchos casos, termina consumiendo y consumiéndose en una lucha desmedida por el confort. Ya no se trata entonces del trabajo como medio de expresión y ratificación en el mundo, sino del trabajo como un fin en sí mismo, por demás, en algunos casos, desprovisto de un sentido distinto a la remuneración económica.

La sociedad actual, además, ha desarrollado una particular existencia y relación con el tiempo: con su percepción, disponibilidad y dificultad, por cierto, cada vez mayor para disponer de éste. Así, se configura una cultura atravesada por la celeridad, lo inmediato y los crecientes insomnios en procura de un éxito que valida sólo el tiempo de la producción intelectiva, laboral o material; relegándose la quietud, el ocio y el disfrute de la vida como aspectos de menor valía para un mundo que se consume en sus desbocados ritmos de progreso.

Sumado a lo anterior, la gran aglomeración de personas en las ciudades ha generado una pluralidad cultural nunca antes vista. En pequeñas "aldeas globales", se presentan profundas tensiones entre los distintos sentidos y relatos que, de antiguo, soportaban y explicaban los "fundamentos estables" del acontecer social, económico, político y cultural. Un escenario de marcada hibridez hace presencia por las amplias avenidas de significación configurando lógicas alternativas, complejas e improbables para modos de pensar enraizados en la predictibilidad que en otros tiempos fundaba lugares seguros a la razón.

Así, algunos "modos de saber" que respondían generalmente a lógicas racionalistas han venido siendo saqueadas por la realidad misma que se fisura ante los nuevos descubrimientos y misteriosos movimientos de la vida en el planeta. Un desbordamiento paradigmático alcanza las artes, ciencias y tecnologías. Nuevas narrativas hacen su aparición en contextos donde lo múltiple y plural encuentra su asidero en manifestaciones creativas y recreativas de la vida misma con altos niveles de afectividad, imaginación y fantasía ${ }^{5}$.

Se hace necesario, entonces, advertir nuevos "podrían ser" explicativos del mundo y sus marcados avatares conducentes a una sociedad distinta que no se agota, necesariamente, en escenarios de trabajo y estudio de marcada racionalización y producción a ultranza de la existencia misma. Sino que, muestra contingencias, intersticios y fugas a arquitecturas po-

5 Al respecto pueden revisarse ideas de Carlos Restrepo (1994). El derecho a la ternura, Bogotá, ed. Reina; Janer, G. Manila (1989). Pedagogía de la imaginación poética, Barcelona, Aliorna; Egan, Kieran (1994) Fantasía e imaginación: su poder en la enseñanza, Madrid, Morata; Vigotsky, L.S. (1982). La imaginación y el arte en la infancia (ensayo psicológico), Madrid, Akal; Ricardo Marín (1984). La creatividad y educación, Barcelona, Ceac; Gardner, Howard (1998). Inteligencias Múltiples, Barcelona, Paidós; Goleman, Daniel (1996). La Inteligencia emocional, Bogotá, Vergara editores; Jiménez Carlos Alberto (1995). Fantasías y Risas, Pereira, Gráficas Olímpica; Krisnamurti, Jiddu (1996). Pedagogía de la Libertad, Barcelona, Integral; Maturana H y Verden Zoller (1995). Amor y Juego, Santiago de Chile, Talleres Gráficos, Pía Sociedad de San Pablo; Popper, Karl (1996). En busca de un mundo mejor, Barcelona, Paidós; entre otras. 
bladas de arte, ocio, juego y creación: situación que no debería ser ajena a la escuela, tratándose de un espacio de "negociación entre realidades" donde la cultura y su multiplicidad de formas expresivas han de constituir un jalonamiento considerable en el progreso de la humanidad.

La escuela enfrenta un protagónico papel en la configuración de un ethos cultural abierto al mundo, a las nuevas sensibilidades de carácter lúdico, conversacional e imaginativo que como bien lo expresaba Novaris, desde la pluma de Rodari (1999), traerán a la escuela una fantástica en la educación: si tuviéramos también una fantástica, como una lógica, habríamos descubierto el arte de inventar. Fantástica, por demás, presente no sólo en el tiempo del recreo, las vacaciones o la fiesta, sino en lo cotidiano y sus distintos lenguajes de la invención.

Según Parra (1996) los aspectos emocionales y el juego han quedado relegados al tiempo corto del recreo:

Los muros de la institución escolar no sólo definen límites entre escuela y comunidad, sino también límites en las formas humana de ser. Afuera de la escuela se queda lo cooperativo natural de la comunidad humana, y a la escuela entra lo competitivo de la fábrica y la guerra. Lo emocional, el juego, la conversación no fácilmente entran en el aula; pareciera que sólo en los recreos existen.

Por lo tanto, contraponer la lúdica a lo serio, el juego al trabajo y al estudio o, la emoción a la razón, es fragmentar aún más la unicidad humana e invalidar estas nuevas narrativas que configuran otro tipo de lógica posible para formar sujetos-maestros del cambio social. Puede argüirse aquí que la educación debe centrar sus esfuerzos para suplir las demandas laborales, sociales o políticas del país. Ello, puede ser cierto. No obstante, la educación no debe ser leída, exclusivamente, como un agenciamientoutilitarista para proveer sujetos-objetos, capaces de insertarse al mundo productivo, del consumo o de las ocupaciones remuneradas. Ha de procurar, abrir "lo posible" para que desde allí un devenir más humano logre advertir que la vida más allá de donde dicen las fábricas, las escuelas y familias que inicia y termina su recorrido.

Dentro de este escenario, se concibe que la recreación y el ocio hacen posible un espacio para pen- sar y dar respuesta, desde la escuela, a una sociedad contemporánea compleja, diversa, impredecible y veloz que demanda entre otras cosas: formas más efectivas de socialización, necesidad de enseñar a manejar el tiempo por parte de los sujetos y dotarlo de sentido, exigentes tareas para permitir integrar la pluralidad cultural a los diferentes círculos sociales, a la impostergable exigencia de comprender críticamente el mundo de los medios de comunicación y sus diferentes lenguajes, a la necesidad de estructurar y generar un repertorio de valores que permitan la interacción entre pueblos y el advenimiento de un sujeto sensible a éticas y estéticas innovadoras.

Y, es precisamente, dentro de esta perspectiva de cambios permanentes y aperturas a las nuevas sensibilidades y narrativas fantásticas del mundo contemporáneo, que la Licenciatura en Recreación concibe a sus maestros en formación centrando su interés en la configuración de, como se dijo antes, un nuevos ethos: cultural creativo, re-creativo, alterativo y transformador de una escuela que como bien lo expresó IIlich (1978) haciendo referencia al insulso tradicionalismo de muchas instituciones, al parecer, ésta es la más perniciosa de todas.

\section{Escuela y sociedad actual}

En la sociedad contemporánea, la escuela, además de educar en conocimientos y/o competencias básicas académicas enfrenta una pregunta fundamental por su pertinencia. Es inaplazable inquirir desde y hacia ella en su papel mediador entre la cultura académica y el mundo; entre lo deseado y lo posible; entre los sueños, aspiraciones y lo que ésta, realmente ofrece. Especialmente, entre los desafíos contemporáneos y los nuevos mapas de sentido para transitar por el mundo. Un mundo por cierto, que experimenta desplazamientos continuos en las distintas esferas, sugiriendo muchos centros, casi ninguna línea recta para encontrarlos: "una esfera espantosa, cuyo centro está en todas partes y la circunferencia en ninguna" (Borges, 1952).

La sociedad contemporánea se ha trasformado a un paso vertiginoso en todas sus dimensiones. Emerge un escenario donde la información regula el quehacer diario y las formas de socialización humana han dejado de ser de naturaleza biológica, emocional e instintiva y dando lugar a una socialización emocionalmente analfabeta: caracterizada 
por la escasa interacción directa, cálida y cordial de la presencialidad. A cambio, una gran interacción entre sujetos virtuales y clickeantes que abren y cierran ventanas comunicativas con desmedido desdén.

Frente a estos escenarios modernos de contingencia marcada en los saberes, la recreación se vislumbra como una opción que hace posible transitar por una sociedad contemporánea, con más centros y menos circunferencias; con más encuentros directos donde el rostro, el contacto y el encuentro son de inevitable tránsito para los sujetos.

\section{Sobre la sociedad contemporánea}

Existen diversas perspectivas de análisis al mundo contemporáneo. Muchas de ellas coinciden en afirmar que como rasgo fundamental, a esos tiempos, el cambio. Diversos autores plantean que se asiste a la construcción de una nueva sociedad: se habla de la sociedad posindustrial o posmoderna, otros la enuncian como la sociedad postradicional, sociedad opulenta, sociedad telemática, sociedad de tercera ola, de segunda ruptura industrial-tecnológica, sociedad red, sociedad digital, sociedad posmaterialista como lo enuncia Águila (2005), ó sociedades líquidas en términos de Bauman (2004).

Se plantea que esta nueva sociedad presenta cambios a nivel tanto tecnológico, cultural, social y político. Afectando ello las formas de relación e intercambio social y cultural. Cornelio Águila, plantea que los cambios contemporáneos tiene sus raíces en las "grandes revoluciones: la americana y la francesa las cuales proporcionaron la estructura política e institucional, y la revolución industrial, que aporto la base económica para el desarrollo del industrialismo, del urbanismo y, finalmente del capitalismo como sistema socioeconómico" (Águila, 2007, p. 13).

Al hacer una comparación entre la sociedad premoderna y posmoderna se evidencia, por ejemplo, cambios en la tecnología. Se pasa de una sociedad pobre en tecnología a una que es avanzada y saturada por el uso de artefactos tecnológicos. De una escasa preparación para el trabajo a una sociedad que especializa su mano de obra, del poco desarrollo de la comunicación y la alfabetización de los sujetos a una alta incidencia en el conocimiento como capital negociador de realidades, de la concepción de pequeñas comunidades como son el barrio, la calle, a las grandes redes interdependientes, de la producción cultural local a la producción de culturas hibridas. Estos, entre otros, son algunos cambios que se han evidenciado en la sociedad. En este sentido interesa detenerse a inquirir en el conocimiento circulante en la sociedad posmoderna, el tipo de relaciones humanas que se instalan en lo cotidiano y la escuela como eje transversal de los aspectos anteriores.

Con relación al conocimiento, (Lyotard, 1987) en su texto La condición posmoderna hace un análisis del saber en las sociedades más desarrolladas, así mismo acuña el término posmoderno, "el cual designa el estado de la cultura después de las transformaciones que han afectado a las reglas de juego de la ciencia, de la literatura y de las artes a partir del siglo XIX". Este autor muestra como entra en crisis los metarrelatos. Así, dice él, pierde sus funciones el gran héroe, los grandes peligros, los grandes periplos y el gran propósito. El papel del conocimiento en esta nueva sociedad es el incremento del poder y la "transparencia comunicacional", es decir, el conocimiento es fuente de ganancias y un medio de control social. Desde esta perspectiva se pasa de los grandes relatos a los pequeños, donde cada sociedad establece narrativas, discursos y racionalidades particulares que no alcanzan, ni pueden constituirse universalmente y que además fluyen con aparente imposibilidad de ocultamiento, de privacidad al sujeto.

En esa misma medida, los cambios políticos y sociales han modificado las formas de relacionarse entre los seres humanos. Algunos autores muestran cómo se entra en la era del consumo en donde no sólo se producen bienes y servicios sino formas de relación. Se adopta un nuevo estilo de vida que como lo enuncia Lipovetsky (2002) "se ha edificado una nueva civilización que ya no se dedica a vencer el deseo sino a exacerbarlo y desculpabilizarlo: los goces del presente, el templo del yo, del cuerpo y de la comodidad se han convertido en la nueva Jerusalén de los tiempos posmoralistas". En este sentido la individualidad, la preocupación por el yo se vuelve cada vez más fuerte. Las relaciones humanas toman otro rumbo: quizás sigue la ensoñación de vivir en comunidad con las libertades de la individualidad, pero, paradójicamente con la imposibilidad de la autenticidad: replicantes y deseantes de un modelo consumista que impone sus estereotipos de seducción a los asiduos fagocitadores del mercado.

La complejidad de estudiar las relaciones humanas contemporáneas pretende, para la escuela, intentar 
comprender cuáles son los principios sobre los que se erigen las relaciones sociales y cómo ésta ha de incidir en nuevas configuraciones de sentido. Si antes se hablaba del compromiso como una categoría determinante, ahora se evidencia su aucencia, lo efímero y los "sin lugares" como aspectos preponderantes para las generaciones que ya no disponen de sus dos manos como antes: una de ellas está, generalmente, pegada al ratón o adicta a algún teclado. Y ni que decir de las redes sociales donde es posible conectarse a ella cuando se quiere pero a su vez partir cuando se desee. Se reconfigura la idea de continuidad y de permanencia advirtiéndose una cultura de pocas estadías y más navegaciones que, a la manera de los buenos surfistas, aman la superficialidad de sus escenarios ya que de ello depende, en buena parte, su deslizamiento.

La cibercultura como lo dice Albornoz (2008) va configurando nuevas nociones del "ser" y novedosas expresiones y modos de representación del sujeto en línea. En este sentido, las relaciones en Internet se constituyen en una nueva estrategia mediacional, que construye prácticas sociales virtuales sincrónicas y asincrónicas, por ejemplo, subir videos, fotografías, el chat, los encuentros virtuales, entre otros. Estas "formas de encuentro" expresan un pensamiento de época cuyas aristas despliegan la presencia de un sujeto mediado, necesariamente, por la virtualidad que ha resolver-ser desde la lógica del nómada posmoderno, por demás, condenado a navegar un espacio sin límites ni caminos visibles de regreso.

Estos aspectos que son parte de la realidad contemporánea permean la escuela. Esta ha de enfrentar desafíos fundamentales frente a las formas de negociación con el conocimiento ${ }^{6}$ y la realidad. Se requiere, con urgencia, sujetos que se formen a lo largo de la vida, flexibles y adaptables a las condiciones inestables del mundo. Es necesario, además, que la escuela reconozca que los aprendizajes de los sujetos fuera de ella son de vital importancia para su progreso. En palabras de Tedesco (2005), las industrias educativas y otras agencias de socialización, como son la empresa, la familia y los medios de comunicación han de contribuir desde una amplia gama de posibilidades educativas a la formación humana.

\footnotetext{
${ }^{6}$ Juan Carlos Tedesco, la explica como una sociedad donde el conocimiento es el eje articulador de la economía y donde su utilización y comercialización es masiva.
}

Desde el punto de vista de Tedesco (2005) la escuela contemporánea ante los cambios evidenciados requiere distinguir tres procesos importantes: "la enseñanza básica, donde el mayor grado de articulación debe establecerse con la familia; el proceso de socialización cultural, donde la articulación más importante se establece con los medios de comunicación; la relación con la empresa, especialmente desde el punto de vista de la formación para el trabajo". Pero a su vez estos tres agentes socializadores se han modificado, la familia no es exactamente la familia tradicional, ha modificado su constitución y formas de relación.

Bauman (2002) plantea como la escuela de hoy debe educar "para que los seres humanos busquen soluciones privadas a los problemas derivados de la sociedad y no soluciones derivadas de la sociedad para los problemas privados". En este sentido, la propuesta por la re-creación ${ }^{7}$ en la escuela llevan a pensar un ser humano creativo, capaz de transformar las realidades de adentro hacia afuera: en la medida que aumenta sus niveles de satisfacción personal podrá incidir como agente transformador para otros.

Por ello es necesario pensar en una escuela que permita la formación de sujetos creativos. Al respecto Parra (1997) advierte:

No se trata de preparar a los sujetos para realizar obras maestras, se espera creadores en el sentido que gestan su singularidad, gestan obras que los representan, se gesta humanamente en cooperación con otros. Se esperan creadores, en el sentido que son creadores que son "aún no" creadores.

Por lo tanto, se espera una escuela que evolucione desde la transformación de las personas, de sus modos de sentir, pensar y expresar su propia singularidad creativa, su potencialidad y recursividad, cuando de vivir se trata.

\section{La creación, recreación y escuela}

En este sentido, la recreación esencialmente es reinvención. Una alternativa creadora, fundadora de "nuevos podrían ser para el mundo". Por lo tanto, es incon-

\footnotetext{
${ }^{7}$ Se entiende por recreación la posibilidad de repensar los procesos estructurados y que pueden sufrir modificaciones gracias al acto creativo e inspirador.
} 
cebible asumir la recreación sin un ejercicio previo y constante de creación humana. La creatividad se erige así, como un elemento fundamental para gestionar la imaginación y desde allí, otorgarle mayores oportunidades al descubrimiento de lo posible.

Desde tiempo atrás la creatividad ha intentado ser desarrollada antes que explicada. Se le ha relacionado con genialidad, inspiración, talento, inconsciencia, bisociación, sublimación. Un halo de misterio ha estado presente frente a su acercamiento: "la propia creatividad es aparentemente un misterio, porque existe en ella algo paradójico, algo que hace difícil de imaginar cómo es tan siquiera posible. Cómo sucede es sin duda enigmático, pero que ocurra es profundamente misterioso"8 (Boden, 1994).

El diccionario define el "crear", en una de sus acepciones, como producir algo de la nada como hizo Dios al dar origen al mundo (Diccionario Norma, 1991). No es de extrañarse que tal situación, de entrada, dificulte la comprensión del crear como un acto cotidiano, realizable frecuentemente por seres mortales incapaces de soplar sobre la nada su aliento para hacer emerger, como en el principio, con la palabra un mundo. Al respecto Boden (1994) nota que las discusiones de los filósofos y teólogos, tiempo atrás, advirtieron la situación paradójica de la creación de la nada (ex nihilo). Proponían que la creación no sólo era por Dios sino a partir de Dios.

Sumado a lo anterior, más adelante en su libro presenta dos enfoques, muy difundidos frente a la creatividad, que han hecho prácticamente imposible su explicación científica: el inspiracional y el romántico.

El enfoque inspiracional ve la creatividad como algo esencialmente misterioso, incluso sobrehumano o divino. Platón lo formula así: 'un poeta es sagrado y nunca es capaz de componer hasta que esté inspirado, poseído, y la razón ya no esté en él [...] porque no soporta el arte que las articula, sino por el poder divino'. El enfoque romántico es menos extremo, ya que afirma que la creatividad-aunque no sea realmente divina es por lo menos excepcional. Se dice que los artistas creativos (y los científicos) son personas dotadas con un talento específico que los otros no poseen: perspicacia e intuición (Bauman, 1994).

${ }^{8}$ Esto es un primer planteamiento de Margaret Boden. No debe llamarse a confusión ello ya que uno de los principales objetivos de la autora es explicar precisamente cómo ocurre el acto creativo desde modelos de procesamiento de información.
Considerar la creatividad como don divino, creación ex nihilo, atributo de algunos pocos científicos o artistas conlleva a estimar determinadas posturas, formativamente hablando al respecto. Por consiguiente, esperar que la "musa de la inspiración" aparezca o centrar la atención en algunos pocos "iluminados" por este don, podrían ser consideradas acciones legitimas dentro de la lógica de estos enfoques inspiracionalistas románticos.

Otra posibilidad es considerar la creatividad como algo inherente a todo ser humano, desarrollable y plausible de explicación científica. Si se considera esta perspectiva, la formación para el desarrollo de la creatividad en contextos educativos deja de ser un misterio y, en consecuencia, debe traducirse en actos humanos creativos que jalonen el desarrollo de la creatividad en otros. Razón que justifica proponer, la creación y re-creación como actos humanamente posibles y además desarrollables en contextos de formación humana.

Jiménez (2004) nota el relevante papel de la Lúdica en la Escuela. Aproxima este saber lúdico como algo fundante a pedagogías del siglo XXI. Su texto desarrolla la importancia de otro tipo de intervenciones y relaciones en la escuela contemporánea, en donde lo simbólico e imaginativo-creador cumple un papel preponderante en la formación de sujetos para el futuro. Por su parte, la propuesta que hace la Universidad de Deusto, muestra como el papel de la pedagogía del ocio es hacer presencia transformadora en la escuela. Cuenca (2004) perfila algunas propuestas para trabajar el ocio, no como algo anexo, de lujo u ocasional, si no como una asignación pedagógica de la escuela del siglo XXI. Planteamientos que, desde las prácticas de ocio, desbordan la institucionalidad escolar alcanzando escenarios sociales y culturales para resarcir el tejido social que se desgasta en estos tiempos de celeridad humana.

Así, la re-creación, de igual modo, se presenta como un importante punto de articulación que le permite al sujeto volver a crear relaciones insospechadas con los objetos y las personas en la escuela. De acuerdo con Mesa (2002) se propone dos tipos de mediación, de naturaleza distinta, frente a la posibilidad de formar desde la recreación ${ }^{9}$, a saber:

\footnotetext{
9 Para ampliar esta idea puede revisarse: Mesa, Guillermina (1993). La recreación dirigida - juego, arte o pedagogía; Documentos Universidad del Valle (2000). ¿La formación Profesio-
} 
1. Lenguajes lúdico-recreativos: constituidas por lo lúdico y por las técnicas recreativas apropiadas del arte (pintura, música, teatro, danza, etc.), la ciencia y la tecnología.

2. El discurso pedagógico: es el lenguaje "instruccional" donde se traman, se entretejen, las normas, las consignas, las instrucciones, las intencionalidades, los acuerdos, las actitudes, las negociaciones, los valores, y en particular los contenidos tanto conceptuales como procedimentales.

Estas dos posibilidades implican, por un lado, fortalecer el discurso hacía una pedagogía de la lúdica, la creatividad y la recreación. Por otra parte, ahondar en lenguajes que propicien el desarrollo de estas posibilidades como estrategia mediacional para transformar la escuela, para su des-fosilización e inserción en las nuevas sensibilidades que conllevan retos tanto en contextos globales como locales. En suma, una escuela capaz de fundar lo posible: académica, estética, creativa y recreativamente desde una miríada de opciones y mediaciones de naturaleza distinta.

\section{Reflexiones finales}

Cajiao (1995) en el proyecto Atlántida, indaga por la relación de la escuela y los jóvenes, dilucidando cómo se entreteje la escuela y sus procesos formativos con la realidad de los jóvenes. Dice que, buscando dar respuesta, la escuela con toda su visión, logra entrecruzar la realidad y la vida de los jóvenes tímidamente con los saberes impartidos desde la tradición académica. En este sentido, Cajiao pregunta: “¿qué tipo de educación corresponde a un mundo como el actual?". El autor, nos ubica frente a un fenómeno que no es de menor importancia e indica que la capacidad de influencia e implicación de la escuela, en el proceso educativo, se ha debilitado de modo notorio siendo relegada por la velocidad de una época que lo desborda, casi todo.

En definitiva, no pueden pensarse que la transformación dinámica de la escuela en procura de una mayor relevancia de su papel en la época actual, se relaciona con cambios superficiales desde políticas de turno o programas coyunturales que desaparecen como si se tratará de una moda más. Se hace

nal en Recreación: un Sueño o una Necesidad Indiscutible?; Documentos Universidad del Valle (1997). La Recreación Dirigida Como Proceso Educativo, Tesis Doctoral, Universidad de Barcelona España. necesario un cambio radical, una transformación genuina frente a objetos, prácticas e imaginarios que han hecho de la escuela una de las instituciones más rezagadas tanto histórica como culturalmente hablando.

No es de sorprenderse que la cultura escolar, ante los problemas referidos líneas atrás, se presente como una propuesta poco atractiva, sesgada, atemporal y desarticulada entre las diferentes realidades que entrecruzan la existencia humana que, por demás, está ávida de un deseo auténtico por la autonomía, la libertad y la creatividad.

Por consiguiente se presenta el saber recreativo como una opción de transformación radical y progresiva en la cultura escolar. Algo deseable para dilucidar en un futuro cercano, escenarios reales para emprender algún sueño $y$, con ello, favorecer la gestión de la imaginación como evento inaplazable ante un mundo que se aún no se ha terminado de descifrar.

\section{Referencias}

Albornoz, M. (2008). 'Ciberculturas y las nuevas nociones de privacidad'. En Revista Nómadas.

Águila, C. (2005). Ocio, Jóvenes y Posmodernidad, España, Universidad de Almería.

Águila, C. (2007) .Sobre el ocio y la posmodernidad: un análisis sociocrítico, Sevilla.

Bauman, Z. (2002). Modernidad Liquida, México, Fondo de Cultura Económica.

Boden, M. (1994). La mente creativa: mitos y mecanismos, Barcelona, Gedisa.

Cajiao, F (1995). Proyecto Atlántida: sobre el adolescente escolar en Colombia, Bogotá, Fundación para la Educación Superior.

Ilich, Iván (2006). Obras reunidas, México, Fondo de cultura económica.

Lyotard, J (1987). La condición posmoderna, Madrid, Ediciones Cátedra.

Lipovetsky, G (2002). El crepúsculo del deber: la ética indolora de los nuevos tiempos, Barcelona, Anagrama.

Mesa, G. (1993). La Recreación Dirigida - Juego, Arte o Pedagogía, Documentos Universidad del Valle.

Parra, J. (1996). Inspiración, Bogotá, Cooperativa Editorial del Magisterio.

Rodari, G. (1999). Gramática de la fantasía, Bogotá, Panamericana.

Sennet, R. (2008). El artesano, Barcelona, Anagrama.

Tedesco, J (2005). Educar en la sociedad del conocimiento, México, Fondo Cultura Económica. 